\title{
Quantitative Gated SPECT-Derived Phase Analysis on Gated Myocardial Perfusion SPECT Detects Left Ventricular Dyssynchrony and Predicts Response to Cardiac Resynchronization Therapy
}

\author{
Mark M. Boogers ${ }^{1,2}$, Serge D. Van Kriekinge ${ }^{3,4}$, Maureen M. Henneman ${ }^{1}$, Claudia Ypenburg ${ }^{1}$, Rutger J. Van Bommel ${ }^{1}$, \\ Eric Boersma ${ }^{5}$, Petra Dibbets-Schneider ${ }^{6}$, Marcel P. Stokkel ${ }^{6}$, Martin J. Schalij ${ }^{1}$, Daniel S. Berman ${ }^{4,7}$, \\ Guido Germano ${ }^{3,4}$, and Jeroen J. Bax ${ }^{1}$ \\ ${ }^{1}$ Department of Cardiology, Leiden University Medical Center, Leiden, The Netherlands; ${ }^{2}$ The Interuniversity Cardiology Institute of \\ The Netherlands, Utrecht, The Netherlands; ${ }^{3}$ Artificial Intelligence in Medicine Program, Cedars-Sinai Medical Center, Los \\ Angeles, California; ${ }^{4}$ David Geffen School of Medicine, UCLA, Los Angeles, California; ${ }^{5}$ Department of Epidemiology and \\ Statistics, Erasmus University, Rotterdam, The Netherlands; ${ }^{6}$ Department of Nuclear Medicine, Leiden University Medical Center, \\ Leiden, The Netherlands; and ${ }^{7}$ Department of Imaging, Cedars-Sinai Medical Center, Los Angeles, California
}

\begin{abstract}
The significance of left ventricular (LV) dyssynchrony for the prediction of response to cardiac resynchronization therapy (CRT) has been demonstrated. Parameters reflecting LV dyssynchrony (phase SD, histogram bandwidth) can be derived from gated myocardial perfusion SPECT (GMPS) using phase analysis. The feasibility of LV dyssynchrony assessment with phase analysis on GMPS using Quantitative Gated SPECT (QGS) software has not been demonstrated in patients undergoing CRT. The aim of the present study was to validate the QGS algorithm for phase analysis on GMPS in a direct comparison with echocardiography using tissue Doppler imaging (TDI) for LV dyssynchrony assessment. Also, prediction of response to CRT using GMPS and phase analysis was evaluated. Methods: Patients $(n=40)$ with severe heart failure (New York Heart Association class III-IV), an LV ejection fraction of no more than $35 \%$, and a QRS complex greater than or equal to 120 ms were evaluated for LV dyssynchrony using GMPS and echocardiography with TDI. At baseline and after 6 mo of CRT, clinical status, LV volumes, and LV ejection fraction were evaluated. Patients with functional improvement were classified as CRT responders. Results: Both histogram bandwidth $\left(r=0.69, r^{2}=0.48\right.$, SEE $=25.4, P<$ $0.01)$ and phase SD $\left(r=0.65, r^{2}=0.42\right.$, SEE $\left.=26.8, P<0.01\right)$ derived from GMPS correlated significantly with TDI for assessment of LV dyssynchrony. At baseline, CRT responders showed a significantly larger histogram bandwidth $\left(94^{\circ} \pm 23^{\circ}\right.$ vs. $68^{\circ} \pm$ $\left.21^{\circ}, P<0.01\right)$ and a larger phase SD $\left(26^{\circ} \pm 6^{\circ}\right.$ vs. $18^{\circ} \pm 5^{\circ}, P<$ 0.01 ) than did nonresponders. Receiver-operating-characteristic curve analysis identified an optimal cutoff value of $72.5^{\circ}$ for histogram bandwidth to predict CRT response, yielding a sensitivity of $83 \%$ and a specificity of $81 \%$. For phase SD, sensitivity and
\end{abstract}

Received Nov. 27, 2008; revision accepted Jan. 29, 2009.

For correspondence or reprints contact: Jeroen J. Bax, Department of Cardiology, Leiden University Medical Center, Albinusdreef 2, 2333 ZA

Leiden, The Netherlands.

E-mail: j.j.bax@lumc.nl

COPYRIGHT @ 2009 by the Society of Nuclear Medicine, Inc. specificity similar to those for histogram bandwidth were obtained at a cutoff value of $19.6^{\circ}$. Conclusion: QGS phase analysis on GMPS correlated significantly with TDI for the assessment of LV dyssynchrony. Moreover, a high accuracy for prediction of response to CRT was obtained using either histogram bandwidth or phase SD.

Key Words: left ventricular dyssynchrony; gated myocardial perfusion SPECT; cardiac resynchronization therapy

J Nucl Med 2009; 50:718-725

DOI: 10.2967/jnumed.108.060657

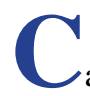

ardiac resynchronization therapy (CRT) has become an established therapeutic option for patients with drugrefractory heart failure. Thus far, several clinical trials have demonstrated the beneficial effect of CRT on symptoms, quality-of-life (QoL) score, and exercise capacity $(1,2)$. In addition, CRT improved left ventricular ejection fraction (LVEF), reduced severity of mitral regurgitation, and decreased left ventricular (LV) volumes (reverse remodeling) $(1,2)$. Moreover, CRT resulted in a superior survival rate, with a reduction in heart failure hospitalization rate $(3,4)$.

Although CRT provides considerable benefit, one third of the patients meeting the current selection criteria do not respond to this form of therapy (5-8), and attention has shifted toward improved patient selection for CRT $(9,10)$. Particularly, observational trials have shown the significance of LV dyssynchrony for the prediction of response to CRT (6-8). LV dyssynchrony can be detected using different imaging modalities, including echocardiography with tissue Doppler imaging (TDI) (6-8) or strain (rate) imaging 
(11), MRI (12), and nuclear imaging with single photon emission computed tomography (SPECT) (13-18). In particular, phase analysis on gated myocardial perfusion SPECT (GMPS) has been evaluated recently for the assessment of LV dyssynchrony (14-18).

Recently, the Quantitative Gated SPECT (QGS) algorithm (Cedars-Sinai) has been expanded to provide quantitative parameters, in addition to the previously reported functional parameters, for the assessment of LV dyssynchrony $(14,18)$. With this algorithm, quantification of LV dyssynchrony is based on phase calculations for each LV surface-sampling point. The aim of the current study was to further validate the QGS-modified algorithm for phase analysis on GMPS in a direct comparison with echocardiography using TDI for the assessment of LV dyssynchrony. Moreover, outcome after 6 mo of CRT was evaluated and related to baseline LV dyssynchrony on GMPS.

\section{MATERIALS AND METHODS}

\section{Study Population and Protocol}

The study population consisted of 40 consecutive drug-refractory heart failure patients who were scheduled for implantation of a CRT device. Eligibility for CRT was based on advanced heart failure (New York Heart Association [NYHA] functional class III-IV), depressed systolic LV function (LVEF $\leq 35 \%$ ), prolonged QRS duration ( $\geq 120 \mathrm{~ms}$ ), and sinus rhythm. Patients with decompensated heart failure, a recent myocardial infarction $(<3 \mathrm{mo})$, or a previously implanted pacemaker were excluded.

Before CRT implantation, all patients underwent evaluation of

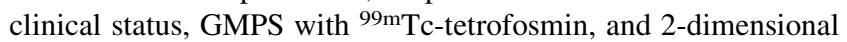
(2D) echocardiography with TDI as part of the clinical assessment to determine therapeutic options.

The clinical status of the patient was assessed on the basis of NYHA functional class, exercise capacity (6-min-walk test), and QoL score (using the Minnesota QoL Questionnaire) (19).

Six months after CRT implantation, assessment of clinical status and 2D echocardiography with TDI were repeated. Patients with an improvement of 1 grade or more in NYHA class were classified as responders to CRT. Patients without improvement in NYHA class were considered nonresponders; patients who died of progressive heart failure were also considered nonresponders.

\section{GMPS}

Data Acquisition. Resting electrocardiogram-gated GMPS with ${ }^{99 \mathrm{~m}} \mathrm{Tc}$-tetrofosmin $(500 \mathrm{MBq}$, injected at rest) was performed with a triple-head SPECT camera system (GCA 9300/HG; Toshiba Corp.). High-resolution, low-energy collimators were used, and a $20 \%$ window was centered around the $140-\mathrm{keV}$ energy peak of ${ }^{99 \mathrm{~m} T c-t e t r o f o s m i n}$. A total of 90 projections (step-and-shoot method, 35 s/projection; $64 \times 64$ matrix; total imaging time, 23 $\min$ ) over a $360^{\circ}$ circular orbit were obtained; data acquisition involved 16 frames per cardiac cycle. Data were reformatted into gated short-axis images by reconstruction with filtered backprojection and reorientation. No attenuation correction was used. Data were displayed in polar map format and analyzed using a 17-segment model (20). Segments with a perfusion defect were defined as those having less than $50 \%$ tracer uptake.

Phase Calculations. These reconstructed images were analyzed by a modified version of the QGS algorithm (21) that provides quantitative parameters, in addition to previously described functional parameters, for the assessment of LV dyssynchrony (14). QGS operates by first computing a mid-myocardial LV surface. The algorithm then computes endo- and epicardial surfaces using count profile and thickening information derived from myocardial mass conservation combined with count increases caused by partial-volume effects. All surfaces are generated as 2D meshes in an ellipsoidal coordinate system, with a fixed number of latitudinal and longitudinal samples (21). The current version of QGS uses 36 longitudinal and 28 latitudinal samples, leading to a maximum of 1,008 sampling points (usually fewer because of surface truncation by the valve plane). Subsequent phase calculations are performed using regional maximal counts obtained by searching between the endocardial and the epicardial surfaces, normally to the mid-myocardial surface, for each surface-sampling point. A unidimensional array is created for each spatial sampling point containing the image counts at each interval. This array represents a time-varying, periodic function to which a cosine curve can be fitted. For this analysis, the constant component can be ignored; therefore, this process is equivalent to reducing the periodic function to its first Fourier harmonic, characterized by an amplitude and phase angle. Once the phase angle has been calculated for each mid-myocardial surface point, a 2D median filter is applied to eliminate spurious variations. The window size of the filter (9 longitudinal samples of 36, 7 latitudinal samples of 28) was selected empirically. Because phase measurements are essentially ill-defined for signals that exhibit low temporal variations (22), 5\% of the samples for which the amplitude is lowest were eliminated.

Dyssynchrony Parameters. Once phase information has been calculated for all sampling points, global and regional dyssynchrony measures can be computed. For this study, a histogram was constructed from the phase values for the entire left ventricle, and phase SD and bandwidth were calculated and examined as the global dyssynchrony measures. Phase SD was calculated in the usual manner. Bandwidth was calculated to include $95 \%$ of histogram elements as previously reported by Chen et al. (15).

Timing values can be expressed in milliseconds ( $\mathrm{ms}$ ), as angles in degrees $\left(0^{\circ}-360^{\circ}\right)$, or as a percentage of the average duration of the cardiac cycle $(0 \%-100 \%)$; the latter 2 values are equivalent. Although timing values expressed in milliseconds have been considered more clinically relevant with echocardiography, they are also directly dependent on the heart rate, potentially rendering the comparison of studies acquired with different heart rates problematic (23). Hence, for this study all timing values are expressed in degrees.

\section{D Echocardiography}

Transthoracic resting 2D echocardiography was performed with a commercially available system (Vivid Seven; General Electric-Vingmed), with the patient placed in a left lateral decubitus position. A $3.5-\mathrm{MHz}$ transducer was used to obtain images at a depth of $16 \mathrm{~cm}$ in the parasternal and apical views (standard long- and short-axis, 2- and 4-chamber images). From apical 2- and 4-chamber images, LV end-systolic (LVESV) and LV end-diastolic (LVEDV) volumes and LVEF were calculated using the biplane Simpson's approach (24). In addition, TDI was performed to assess LV dyssynchrony. For TDI (triggered to the QRS complex), color Doppler frame rates exceeded 80 frames/s, and pulse repetition frequencies varied between $500 \mathrm{~Hz}$ and $1 \mathrm{kHz}$, yielding aliasing velocities between $16 \mathrm{~cm} / \mathrm{s}$ and $32 \mathrm{~cm} / \mathrm{s}$. 
Tissue Doppler images and conventional echo images were saved in cine-loop format and analyzed by 2 experienced observers unaware of the GMPS and clinical patient data. All analyses were performed using commercially available software (Echopac 6.0.1; General Electric-Vingmed). To determine LV dyssynchrony, the sample volume was placed in the basal portions of the septum and lateral wall; peak systolic velocities and time-topeak systolic velocities were obtained, and the delay in peak systolic velocity between the septum and the lateral wall was calculated as an indicator of LV dyssynchrony (25). Reliable tissue Doppler curves for the assessment of septal-to-lateral delay were obtained in 39 patients.

The peak systolic velocity analysis was limited to the LV ejection phase (marked by the opening and closing of the aortic valve). The inter- and intraobserver agreements for assessment of LV dyssynchrony were $90 \%$ and $96 \%$, respectively (26). Substantial LV dyssynchrony on TDI was defined as a delay in peak systolic velocity greater than or equal to $65 \mathrm{~ms}$ between the basal septum and the lateral wall (7).

\section{CRT Implantation}

The LV pacing lead was inserted transvenously via the subclavian route as reported previously (25). A coronary sinus venogram was obtained during occlusion of the coronary sinus using a balloon catheter. The LV pacing lead was inserted into the coronary sinus with an 8-French guiding catheter and positioned as far as possible in the venous system, preferably in the posterolateral vein. The right atrial and right ventricular leads were positioned conventionally. For each patient, the atrioventricular interval was adjusted to maximize the mitral inflow duration using pulsedwave Doppler echocardiography. No adjustments were made to the V-V interval during the first 6 mo of CRT. The CRT-device and LV-lead implantations were successful in all patients, and there were no major complications. All patients received a CRT-D device (Contak Renewal [Guidant]; InSync III, InSync Marquis, or InSync Sentry [Medtronic Inc.]; Atlas HF [St. Jude Medical]), and 4 types of pacemaker leads were used (Acuity 4554 or Easytrak 4517-4524 [Guidant]; Attain 4193-4194 [Medtronic]; Quicksite 1056T [St. Jude Medical]).

\section{Statistical Analysis}

Continuous data are expressed as mean $\pm \mathrm{SD}$, and categoric data are presented as percentages. When appropriate, the 2-tailed paired or unpaired Student $t$ test and the $\chi^{2}$ test were used for numeric or categoric data.

The relationship between histogram bandwidth and phase SD derived from GMPS and LV dyssynchrony derived from TDI was evaluated by Pearson correlation analysis. Next, univariate and multivariate analyses were performed to determine predictors of response to CRT. Receiver-operating-characteristic (ROC) curves were analyzed to determine the optimal cutoff values of histogram bandwidth and phase SD to predict response to CRT. The highest sum of sensitivity and specificity was considered as the optimal cutoff value for the prediction of response to CRT.

For all analyses, a $P$ value of less than 0.05 was considered statistically significant.

\section{RESULTS}

\section{Study Population}

Baseline characteristics of the 40 patients (32 men; mean age, $63 \pm 8 \mathrm{y}$ ) are summarized in Table 1 . The etiology of

\begin{tabular}{|c|c|}
\hline Baseline characteristic & Data \\
\hline Age (y) & $63 \pm 8$ \\
\hline Sex (M/F) & $32 / 8$ \\
\hline Ischemic cardiomyopathy & 28 (70\%) \\
\hline Idiopathic dilated cardiomyopathy & $12(30 \%)$ \\
\hline NYHA functional class & $3.1 \pm 0.4$ \\
\hline QoL score & $38 \pm 19$ \\
\hline 6-min-walk distance $(\mathrm{m})$ & $323 \pm 96$ \\
\hline QRS duration (ms) & $156 \pm 34$ \\
\hline LVEDV (mL) & $252 \pm 84$ \\
\hline LVESV (mL) & $193 \pm 79$ \\
\hline LVEF (\%) & $25 \pm 8$ \\
\hline Cardiac segments with perfusion defect & $6 \pm 3$ \\
\hline \multicolumn{2}{|c|}{$\begin{array}{l}\text { Data are represented as mean } \pm \text { SD or number, with } \\
\text { percentages in parentheses. } \\
\text { NYHA = New York Heart Association; QoL = quality of life; } \\
\text { LVEDV = left ventricular end-diastolic volume; LVESV = left } \\
\text { ventricular end-systolic volume; LVEF = left ventricular ejection } \\
\text { fraction. }\end{array}$} \\
\hline
\end{tabular}

heart failure was ischemic cardiomyopathy in $70 \%$ of patients and idiopathic dilated cardiomyopathy in $30 \%$. Medication consisted of diuretics ( $90 \%$ of patients), angiotensin-converting enzyme inhibitors ( $90 \%$ of patients), and $\beta$-blockers ( $75 \%$ of patients) in maximum tolerated dosages. Before CRT implantation, the mean NYHA functional class was $3.1 \pm 0.4$ and the mean QoL score was $38 \pm$ 19. On the surface electrocardiogram, the mean QRS interval was $156 \pm 34 \mathrm{~ms}$, and the 6-min-walk distance was $323 \pm 96 \mathrm{~m}$. On 2D echocardiography, the mean LVESV was $193 \pm 79 \mathrm{~mL}$, with a mean LVEDV of $252 \pm$ $84 \mathrm{~mL}$ and a mean LVEF of $25 \% \pm 8 \%$. Mean heart rate during GMPS was $68.6 \pm 12.9$ beats $/ \mathrm{min}$, and the mean heart rate during TDI was $68.5 \pm 13.2$ beats/min $(P=$ not significant). The mean number of cardiac segments with a perfusion defect was $6 \pm 3$.

\section{Dyssynchrony: GMPS Versus TDI}

Phase analysis on GMPS yielded a mean histogram bandwidth of $84^{\circ} \pm 26^{\circ}$ (range, $40^{\circ}-152^{\circ}$ ) and a mean phase SD of $23^{\circ} \pm 7^{\circ}\left(\right.$ range, $\left.12^{\circ}-41^{\circ}\right)$. On TDI, the mean LV dyssynchrony was $53 \pm 35 \mathrm{~ms}$ (range, 0-130 ms). Pearson correlation showed a significant correlation between LV dyssynchrony on TDI and histogram bandwidth on GMPS $\left(r=0.69, r^{2}=0.48\right.$, SEE $=25.4, n=39, P<$ 0.01, Fig. 1A). Also, a significant correlation between LV dyssynchrony on TDI and phase SD on GMPS was demonstrated $\left(r=0.65, r^{2}=0.42, \mathrm{SEE}=26.8, n=39, P<\right.$ 0.01 , Fig. 1B). Finally, a good correlation between histogram bandwidth and phase SD was observed $(r=0.95$, $r^{2}=0.91$, SEE $\left.=8.0, P<0.01\right)$.

Patients with LV dyssynchrony greater than or equal to $65 \mathrm{~ms}$ on TDI (indicating substantial LV dyssynchrony) had significantly larger histogram bandwidth and phase SD than 

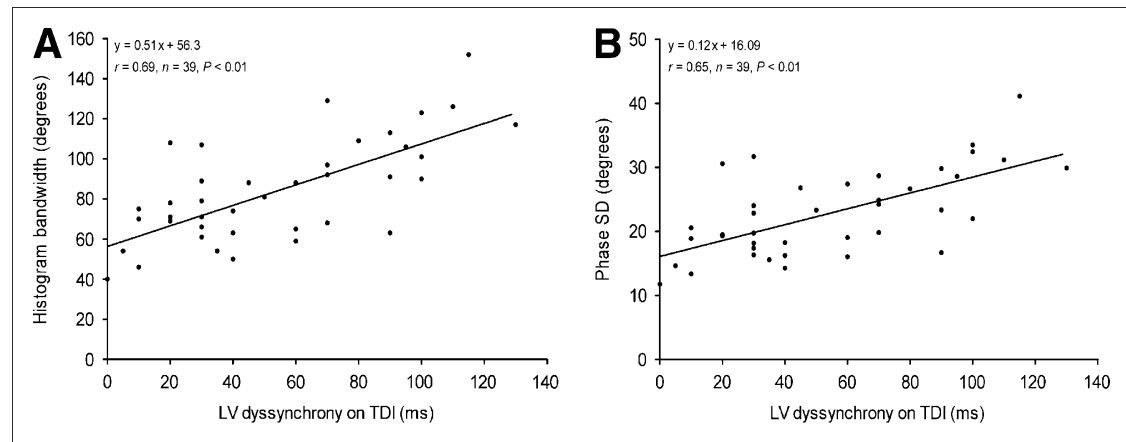

FIGURE 1. Agreement between LV dyssynchrony on TDI and histogram bandwidth $(A)$ or phase SD (B) derived from GMPS. Significant correlation between LV dyssynchrony on TDI and histogram bandwidth or phase SD as assessed with GMPS was observed.

did patients with LV dyssynchrony below $65 \mathrm{~ms}$ on TDI (Fig. 2).

\section{Clinical Responders and Nonresponders}

At the 6-mo follow-up, 24 (60\%) patients showed an improvement of 1 or more grades in NYHA functional class and were considered responders to CRT. Among the 16 (40\%) nonresponders, 5 patients died within 6 mo of CRT because of progressive heart failure.

In Table 2, baseline clinical and imaging variables for responders and nonresponders are summarized. Responders showed a significantly longer 6-min-walk distance and larger LVEDV. Furthermore, responders had significantly more LV dyssynchrony on TDI (Fig. 3).

For the GMPS data, responders showed significantly larger histogram bandwidth $\left(94^{\circ} \pm 23^{\circ}\right.$ vs. $68^{\circ} \pm 21^{\circ}, P<$ $0.01)$ and phase $\mathrm{SD}\left(26^{\circ} \pm 6^{\circ}\right.$ vs. $\left.18^{\circ} \pm 5^{\circ}, P<0.01\right)$ than did nonresponders. Responders to CRT showed a significantly lower number of cardiac segments with perfusion defects than did nonresponders to CRT ( $5 \pm 2$ vs. $8 \pm 2$, $P<0.01)$. Examples of GMPS data in patients with and without response to CRT are shown in Figure 4.

\section{Baseline and 6-Mo Follow-up Data}

At the 6-mo follow-up, responders showed a significant improvement in NYHA functional class, QoL score, and 6-min-walk distance, whereas nonresponders did not show significant improvement in any of the clinical parameters (Table 3). Furthermore, at the 6-mo follow-up only re- sponders demonstrated a significant decrease in LVEDV and LVESV and a significant increase in LVEF.

Univariate analysis was performed on clinical, echocardiographic, and GMPS variables and demonstrated that 6-min-walk distance, LVEDV, and LV dyssynchrony on TDI were predictors of response to CRT at the 6-mo follow-up. In addition, histogram bandwidth and phase SD were predictive of CRT response at the 6-mo follow-up. Multivariate analysis showed that 6-min-walk distance $(P=0.042)$ and phase SD $(P<0.01)$ were the only independent predictors for response to CRT.

ROC curve analysis was used to define the optimal threshold for prediction of response to CRT. For histogram bandwidth, optimal cutoff value was defined at $72.5^{\circ}$, which yielded a sensitivity of $83 \%$ and a specificity of $81 \%$; the area under the curve (AUC) was 0.83 (Fig. 5A). For phase $\mathrm{SD}$, optimal cutoff value was defined at $19.6^{\circ}$, yielding a sensitivity of $83 \%$ and a specificity of $81 \%$; the AUC was 0.85 (Fig. 5B).

\section{DISCUSSION}

Phase analysis on GMPS and echocardiography using TDI showed a significant correlation for the assessment of LV dyssynchrony. Moreover, both histogram bandwidth and phase SD derived from GMPS were predictive of CRT response at the 6-mo follow-up. For histogram bandwidth, an optimal cutoff value of $72.5^{\circ}$ yielded a sensitivity of $83 \%$ and a specificity of $81 \%$ to predict response to CRT.
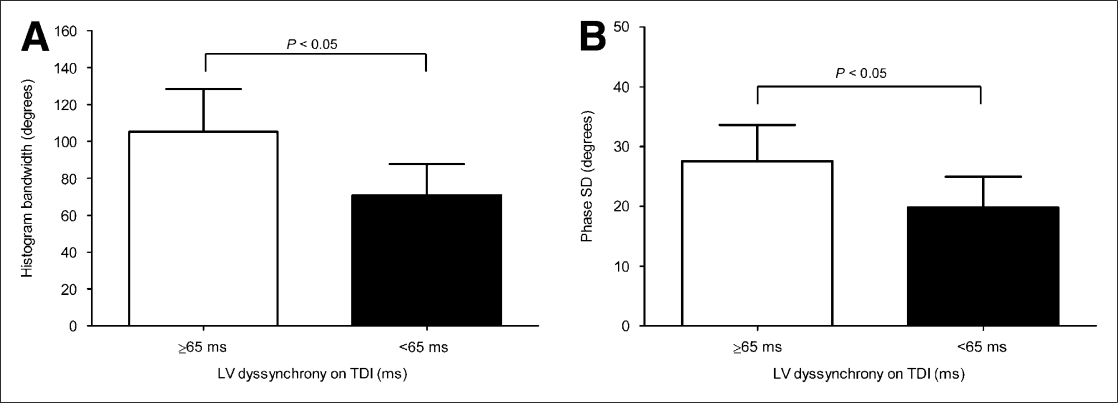

FIGURE 2. (A) Histogram bandwidth was significantly larger in patients with substantial LV dyssynchrony on TDI (defined as mechanical delay between basal septum and lateral wall $\geq 65 \mathrm{~ms}$ ) than that in patients without LV dyssynchrony on TDI (delay $<65 \mathrm{~ms})(105 \pm 23$ vs. $71 \pm 17$, $P<0.05)$. (B) Phase SD was significantly larger in patients with substantial LV dyssynchrony on TDI (defined as mechanical delay between basal septum and lateral wall $\geq 65 \mathrm{~ms}$ ) than that in patients without LV dyssynchrony on TDI (delay $<65 \mathrm{~ms})(28 \pm 6$ vs. $20 \pm 5, P<0.05)$. 
TABLE 2. Baseline Characteristics of Clinical Responders and Nonresponders

\begin{tabular}{|c|c|c|c|}
\hline $\begin{array}{c}\text { Baseline } \\
\text { characteristic }\end{array}$ & $\begin{array}{l}\text { Responders } \\
\qquad(n=24)\end{array}$ & $\begin{array}{l}\text { onresponders } \\
(n=16)\end{array}$ & $P$ \\
\hline Age (y) & $62 \pm 8$ & $64 \pm 9$ & NS \\
\hline $\mathrm{M} / \mathrm{F}$ & $20 / 4$ & $12 / 4$ & NS \\
\hline Ischemic etiology & $16(67)$ & $12(75)$ & NS \\
\hline \multicolumn{4}{|l|}{ Clinical evaluation } \\
\hline NYHA class & $3.0 \pm 0.2$ & $3.2 \pm 0.5$ & NS \\
\hline QoL score & $34 \pm 17$ & $45 \pm 20$ & NS \\
\hline 6-MWT (m) & $354 \pm 81$ & $272 \pm 99$ & 0.01 \\
\hline QRS (ms) & $162 \pm 35$ & $146 \pm 31$ & NS \\
\hline \multicolumn{4}{|l|}{$\begin{array}{l}\text { 2D echocardiographic } \\
\text { parameters }\end{array}$} \\
\hline LVEDV (mL) & $274 \pm 79$ & $219 \pm 83$ & 0.04 \\
\hline LVESV (mL) & $211 \pm 75$ & $167 \pm 78$ & NS \\
\hline LVEF (\%) & $24 \pm 8$ & $25 \pm 8$ & NS \\
\hline LV dyssynchrony (ms) & $62 \pm 37$ & $40 \pm 26$ & 0.04 \\
\hline \multicolumn{4}{|l|}{ Phase distribution } \\
\hline Bandwidth $\left({ }^{\circ}\right)$ & $94 \pm 23$ & $68 \pm 21$ & $<0.01$ \\
\hline Phase SD $\left(^{\circ}\right)$ & $26 \pm 6$ & $18 \pm 5$ & $<0.01$ \\
\hline $\begin{array}{l}\text { Cardiac segments with } \\
\text { perfusion defect }\end{array}$ & $5 \pm 2$ & $8 \pm 2$ & $<0.01$ \\
\hline \multicolumn{4}{|c|}{$\begin{array}{l}\text { Data are represented as mean } \pm \text { SD or number, with } \\
\text { percentages in parentheses. } \\
\text { NS = not significant; NYHA = New York Heart Association; } \\
\text { QoL = quality of life; 6-MWT }=6 \text {-min walk test; } 2 \mathrm{D}=2 \text { - } \\
\text { dimensional; LVEDV = left ventricular end-diastolic volume; } \\
\text { LVESV = left ventricular end-systolic volume; LVEF = left } \\
\text { ventricular ejection fraction; SD = standard deviation. }\end{array}$} \\
\hline
\end{tabular}

For phase SD, sensitivity and specificity similar to those for histogram bandwidth were obtained at a cutoff value of $19.6^{\circ}$.

\section{Dyssynchrony Derived from GMPS}

Patients are selected for CRT on the basis of the current American Heart Association/American College of Cardiology/European Society of Cardiology guidelines, which recommend CRT in patients with NYHA class III or IV heart

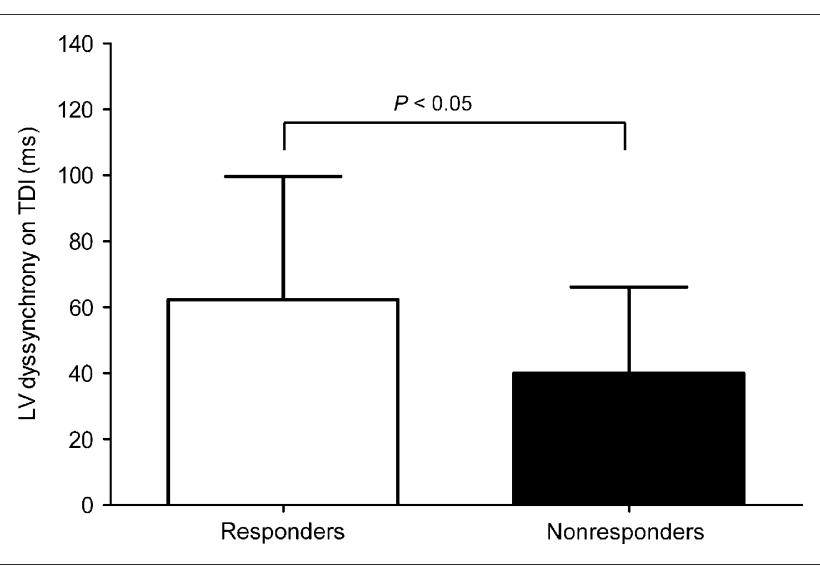

FIGURE 3. Extent of LV dyssynchrony on TDI at baseline was significantly larger in responders to CRT than that in nonresponders to CRT (62 \pm 37 vs. $40 \pm 26, P<0.05)$. failure, an LVEF of no more than 35\%, and a prolonged QRS interval greater than or equal to $120 \mathrm{~ms}(6-10)$.

However, a substantial number of the selected patients do not show response to CRT $(2,5-8)$. Therefore, efforts have been made to improve current selection criteria for CRT, and previous studies have demonstrated that LV dyssynchrony is related to CRT response and may have additional value in the selection of potential candidates for CRT (6-8). One of the potential explanations for nonresponse in patients meeting contemporary guidelines is that a wide QRS complex may reflect interventricular rather than intraventricular dyssynchrony (26).

To detect LV dyssynchrony, a variety of imaging techniques can be used, including echocardiography with TDI (6-8) or myocardial strain (rate) imaging (11), MRI (12), and nuclear imaging with GMPS (14-18). Most studies on CRT have reported on echocardiographic methods to assess LV dyssynchrony; in particular, echocardiography using TDI has been used as a tool for the assessment of LV dyssynchrony (6-8). Recently, the feasibility of phase analysis on GMPS for detection of LV dyssynchrony has been shown $(27,28)$. This approach provides automatically quantitative parameters of LV dyssynchrony using countbased analysis and has been developed to extract amplitude and phase angle from changes in counts per region during the cardiac cycle (14-17). Amplitude of the phase curve indicates maximal systolic wall thickening, whereas phase angle indicates the beginning of mechanical contraction per region, providing information on the degree of hetero- or homogeneity of the mechanical activation pattern within the ventricle. A histogram was constructed that represents the distribution of the phase angle for all sample points of the left ventricle, providing quantitative parameters for LV dyssynchrony including histogram bandwidth (includes $95 \%$ of the elements of the phase histogram) and phase SD (SD of phase distribution) (18).

Various studies have shown that this approach can detect LV dyssynchrony in patients with heart failure and a wide QRS complex $(27,28)$. Subsequent studies have shown the relationship between phase analysis on GMPS and echocardiographic techniques for the assessment of LV dyssynchrony $(29,30)$. Henneman et al. (29) evaluated 75 heart failure patients with a wide QRS complex in a head-to-head comparison between phase analysis on GMPS and 2D echocardiography with TDI. The authors demonstrated that histogram bandwidth and phase SD derived from GMPS related well to LV dyssynchrony derived from TDI. Marsan et al. (30) used real-time 3-dimensional echocardiography and also demonstrated a good relationship with phase analysis on GMPS for the detection of LV dyssynchrony in 40 patients with heart failure. In the current study, GMPS data were processed with the QGS algorithm, which was expanded to provide quantitative parameters for the assessment of LV dyssynchrony. It was shown that LV dyssynchrony derived from TDI related significantly to histogram bandwidth and phase SD obtained with the QGS algorithm. 


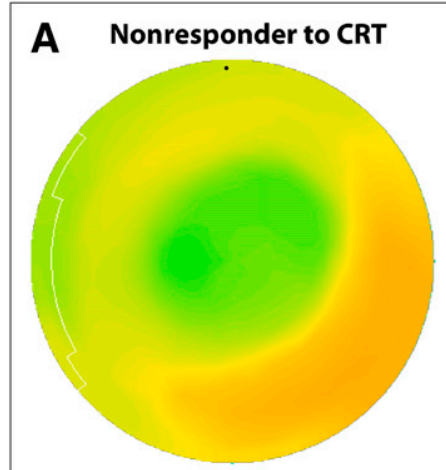

LV counts phase angle polar map

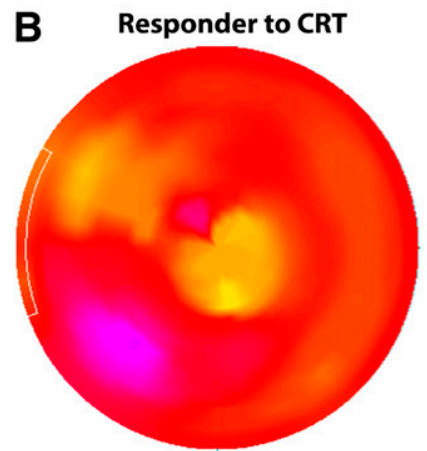

LV counts phase angle polar map

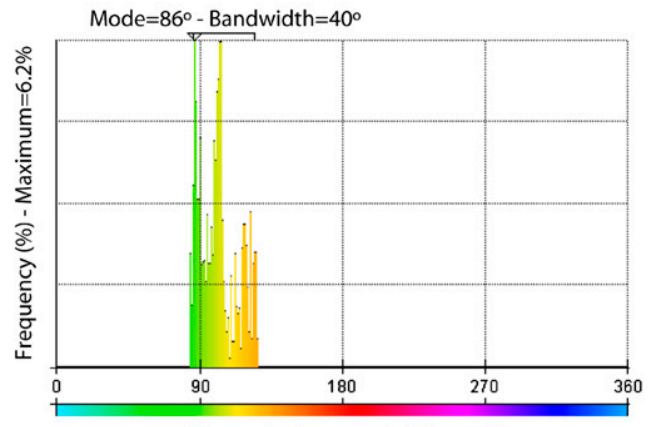

LV counts phase angle (degrees)

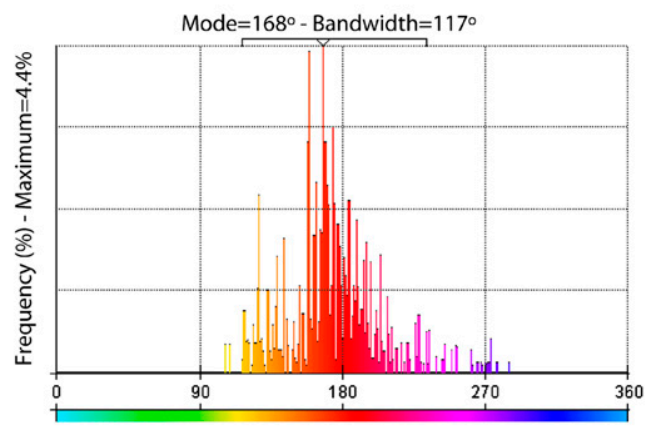

LV counts phase angle (degrees)
FIGURE 4. (A) Example of a patient without LV dyssynchrony on GMPS. Synchronous contraction pattern is reflected by homogeneous phase angle distribution of polar map (left) and narrow highly peaked histogram (right). At 6-mo follow-up, no improvement in NYHA functional class was observed and LVEF remained unchanged $(32 \%$ at baseline vs. $33 \%$ at $6-$ mo follow-up). (B) Example of a patient with extensive LV dyssynchrony on GMPS. LV dyssynchrony is indicated by heterogeneous phase angle distribution of polar map (left) and wide histogram (right). At 6-mo follow-up, NYHA functional class improved from 3 to 2 , with increase in LVEF from $21 \%$ to $33 \%$.

\section{GMPS for Prediction of Response to CRT}

Many echocardiographic studies have shown that LV dyssynchrony is predictive for response to CRT (6-8). Only 1 study has reported on the use of GMPS for prediction of response to CRT (31); in that study, 42 patients with heart failure (NYHA class III-IV), an LVEF of no more than $35 \%$, and a QRS greater than 120 ms underwent GMPS before CRT implantation. In the patients, reflectors of LV dyssynchrony (phase SD and histogram bandwidth) were identified as predictors of response to CRT over a 6-mo follow-up period. Particularly, a cutoff value of $43^{\circ}$ for phase SD yielded a sensitivity and specificity of $74 \%$ for prediction of response to CRT; a cutoff value of $135^{\circ}$ for histogram bandwidth had a sensitivity and specificity of $70 \%$.

In the current study, a different algorithm derived from the QGS program was used for the assessment of phase SD and histogram bandwidth. In line with previous work (31), histogram bandwidth and phase SD were significantly larger in CRT responders than in CRT nonresponders (Table 2). When ROC curve analysis was applied, a cutoff value of $19.6^{\circ}$ for phase SD yielded a sensitivity of $83 \%$ and a specificity of $81 \%$ (AUC, 0.85 ) to predict response to CRT at the 6-mo follow-up. For histogram bandwidth, a similar sensitivity and specificity were obtained at a cutoff value of $72.5^{\circ}$ (AUC, 0.83). The accuracy to predict response to CRT in the current study is in line with earlier work using different software to analyze GMPS (31). The optimal cutoff values for phase SD and histogram bandwidth in the present study are not identical to the values obtained in the study by Henneman et al. (31), and this may be related to differences in software packages or to differences in study populations. One of the major differences between these software packages is that QGS uses an ellipsoidal sampling system (21), whereas the Emory Cardiac Toolbox uses the hybrid cylindric-spheric coordinate system (32).

In both the study by Henneman et al. (31) and the current study, the accuracy of GMPS to predict response to CRT was not perfect. Compared with TDI, however, the advantages of GMPS include providing global measurements and having measurements that are automatically derived. Given these advantages and the accuracy demonstrated by this and previous work, GMPS may provide a reasonable alternative for clinical use to TDI in predicting CRT response.

It is not realistic to expect a perfect accuracy for prediction of CRT response based solely on LV dyssynchrony. Indeed, recent work has indicated that issues other than LV dyssynchrony are important for response to CRT. Scar tissue has been identified as an important factor limiting the response to CRT $(33,34)$. It has been shown that the extent of scar tissue was inversely related to the response to CRT; also, patients with scar tissue in the region where the LV lead was positioned did not respond well to CRT and the extent (the total scar burden) of scar tissue has been shown to prevent response to CRT. Therefore, the preferred method would be to assess not only the presence of LV dyssynchrony but also the extent and location of scar tissue in the left ventricle. In this perspective, GMPS has the 
TABLE 3. Baseline and 6-Month Follow-up Characteris-

tics: Responders Versus Nonresponders

\begin{tabular}{|c|c|c|c|}
\hline Characteristic & $\begin{array}{l}\text { Responders } \\
\quad(n=24)\end{array}$ & $\begin{array}{l}\text { Nonresponders } \\
\quad(n=16)\end{array}$ & $P$ \\
\hline \multicolumn{4}{|l|}{ NYHA class } \\
\hline Baseline & $3.0 \pm 0.2$ & $3.2 \pm 0.5$ & NS \\
\hline Follow-up & $1.7 \pm 0.5^{\star}$ & $3.2 \pm 0.5$ & $<0.01$ \\
\hline \multicolumn{4}{|l|}{ QoL score } \\
\hline Baseline & $34.4 \pm 17.4$ & $44.9 \pm 20.4$ & NS \\
\hline Follow-up & $21.6 \pm 19.2^{*}$ & $40.0 \pm 16.3$ & $<0.05$ \\
\hline \multicolumn{4}{|l|}{ 6-MWT (m) } \\
\hline Baseline & $353.9 \pm 80.9$ & $272.2 \pm 99.4$ & $<0.05$ \\
\hline Follow-up & $439.8 \pm 102.9^{*}$ & $326.8 \pm 108.6$ & $<0.05$ \\
\hline \multicolumn{4}{|l|}{ LVEDV (mL) } \\
\hline Baseline & $274.4 \pm 79.2$ & $219.3 \pm 82.8$ & $<0.05$ \\
\hline Follow-up & $243.7 \pm 77.0^{\star}$ & $180.7 \pm 56.5$ & $<0.05$ \\
\hline \multicolumn{4}{|l|}{ LVESV (mL) } \\
\hline Baseline & $210.5 \pm 75.4$ & $167.1 \pm 78.4$ & NS \\
\hline Follow-up & $166.8 \pm 76.8^{*}$ & $129.3 \pm 50.1$ & NS \\
\hline \multicolumn{4}{|l|}{ LVEF (\%) } \\
\hline Baseline & $23.9 \pm 7.5$ & $25.4 \pm 8.1$ & NS \\
\hline Follow-up & $33.8 \pm 12.7^{*}$ & $30.0 \pm 8.1$ & NS \\
\hline \multicolumn{4}{|c|}{ LV dyssynchrony (ms) } \\
\hline Baseline & $62.3 \pm 37.4$ & $40.0 \pm 26.1$ & $<0.05$ \\
\hline Follow-up & $40.4 \pm 32.5$ & $33.2 \pm 31.6$ & NS \\
\hline $\begin{array}{l}{ }^{*} P<0.05 \text { follow- } \\
\text { Data are represe } \\
\text { with percentages in } \\
N S=\text { not signific }\end{array}$ & $\begin{array}{l}\text { vs. baseline. } \\
\text { d as mean } \pm \mathrm{S} \\
\text { rentheses. } \\
\text {; other abbreviat }\end{array}$ & SD or absolute & numbe \\
\hline
\end{tabular}

potential to provide both these parameters from the same dataset.

Finally, it has been shown that patients with the LV lead positioned outside the region of latest mechanical activation benefitted less from CRT than did patients with the lead positioned in the area of latest activation (35). More recently, Ypenburg et al. (36) reported on the outcome of patients undergoing CRT with the LV lead positioned inside or outside the region of latest mechanical activation and demonstrated that long-term survival was better when the LV pacing lead was positioned at the site of latest mechanical activation.

\section{Study Limitations}

The current work should be considered a feasibility study, validating a new algorithm on phase analysis with QGS. Patients with extensive scar tissue in the left ventricle benefit less from CRT. In addition, patients with the LV pacing lead located outside the region of latest mechanical activation will have a less favorable response to CRT. In the current study, only the number of cardiac segments with a perfusion defect was integrated. To provide a meaningful analysis on all these issues, however, a much larger study population is needed. Whether integration of all this information will result in a superior prediction of response to CRT needs further testing in future studies.

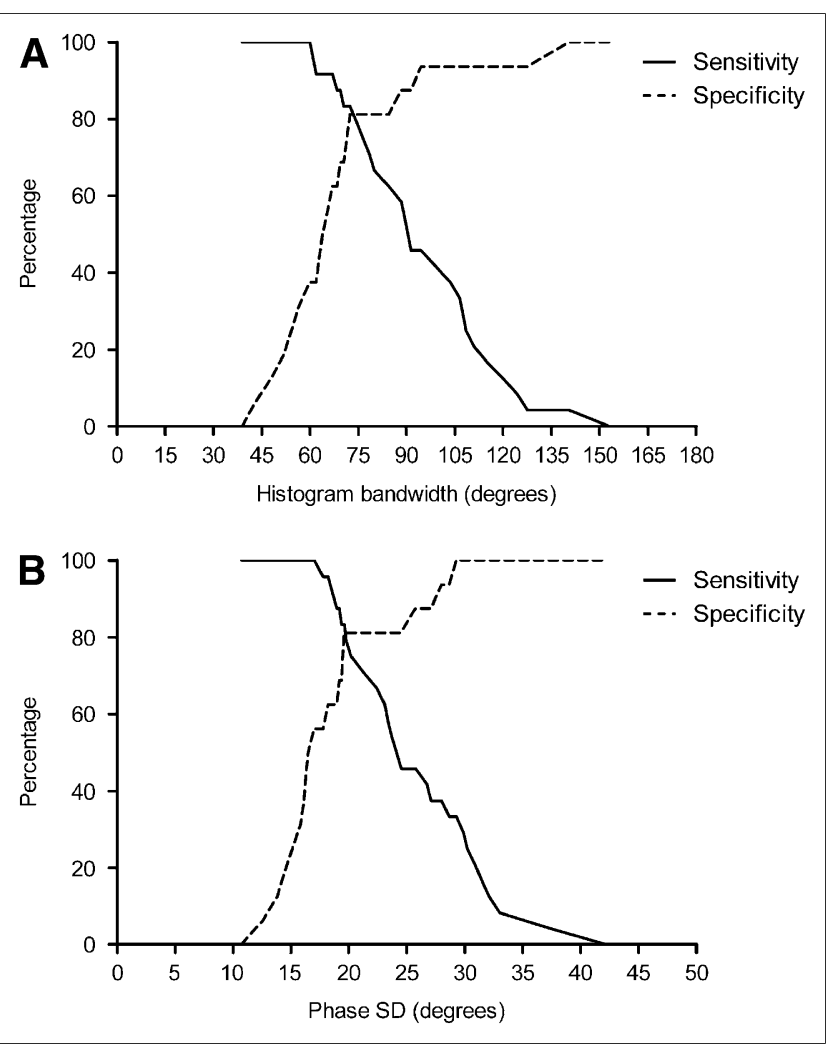

FIGURE 5. ROC curve analysis for histogram bandwidth (A) and phase SD (B) for prediction of response to CRT. ROC curve analysis for histogram bandwidth showed good predictive value (AUC, 0.83) of response to CRT. Optimal cutoff value was defined at $72.5^{\circ}$, yielding sensitivity of $83 \%$ and specificity of $81 \%$. For phase SD, ROC curve analysis demonstrated good predictive value (AUC, 0.85) of response to CRT. Optimal cutoff value was defined at $19.6^{\circ}$, yielding sensitivity of $83 \%$ and specificity of $81 \%$.

\section{CONCLUSION}

Phase analysis on GMPS using QGS software correlated significantly with TDI for the assessment of LV dyssynchrony. In addition, both phase SD and histogram bandwidth were predictive of response to CRT.

Larger studies are needed to confirm these findings and to explore the relative clinical effectiveness of GMPS and TDI in prediction of response to CRT.

\section{ACKNOWLEDGMENTS}

A portion of this study was supported by the Dutch Heart Foundation grant 2006T102. Cedars-Sinai Medical Center receives royalties from the licensing of QGS.

\section{REFERENCES}

1. Cazeau S, Leclercq C, Lavergne T, et al. Effects of multisite biventricular pacing in patients with heart failure and intraventricular conduction delay. $N$ Engl J Med. 2001;344:873-880.

2. Abraham WT, Fisher WG, Smith AL, et al. Cardiac resynchronization in chronic heart failure. N Engl J Med. 2002;346:1845-1853. 
3. Cleland JG, Daubert JC, Erdmann E, et al. The effect of cardiac resynchronization on morbidity and mortality in heart failure. N Engl J Med. 2005;352:1539-1549.

4. Bristow MR, Saxon LA, Boehmer J, et al. Cardiac-resynchronization therapy with or without an implantable defibrillator in advanced chronic heart failure. N Engl J Med. 2004;350:2140-2150.

5. Bax JJ, Van der Wall EE, Schalij MJ. Cardiac resynchronization therapy for heart failure. N Engl J Med. 2002;347:1803-1804.

6. Yu CM, Fung WH, Lin H, Zhang Q, Sanderson JE, Lau CP. Predictors of left ventricular reverse remodeling after cardiac resynchronization therapy for heart failure secondary to idiopathic dilated or ischemic cardiomyopathy. Am J Cardiol. 2003;91:684-688.

7. Bax JJ, Bleeker GB, Marwick TH, et al. Left ventricular dyssynchrony predicts response and prognosis after cardiac resynchronization therapy. J Am Coll Cardiol. 2004;44:1834-1840.

8. Bax JJ, Abraham T, Barold SS, et al. Cardiac resynchronization therapy: part 1issues before device implantation. J Am Coll Cardiol. 2005;46:2153-2167.

9. Hunt SA, Abraham WT, Chin MH, et al. ACC/AHA 2005 guideline update for the diagnosis and management of chronic heart failure in the adult: a report of the American College of Cardiology/American Heart Association Task Force on Practice Guidelines (Writing Committee to Update the 2001 Guidelines for the Evaluation and Management of Heart Failure)—developed in collaboration with the American College of Chest Physicians and the International Society for Heart and Lung Transplantation: endorsed by the Heart Rhythm Society. Circulation. 2005;112:e154-e235.

10. Swedberg K, Cleland J, Dargie H, et al. Guidelines for the diagnosis and treatment of chronic heart failure: executive summary-the Task Force for the Diagnosis and Treatment of Chronic Heart Failure of the European Society of Cardiology. Eur Heart J. 2005;26:1115-1140.

11. Delgado V, Ypenburg C, van Bommel RJ, et al. Assessment of left ventricular dyssynchrony by speckle tracking strain imaging comparison between longitudinal, circumferential, and radial strain in cardiac resynchronization therapy. J Am Coll Cardiol. 2008;51:1944-1952.

12. Westenberg JJ, Lamb HJ, van der Geest RJ, et al. Assessment of left ventricular dyssynchrony in patients with conduction delay and idiopathic dilated cardiomyopathy: head-to-head comparison between tissue Doppler imaging and velocityencoded magnetic resonance imaging. J Am Coll Cardiol. 2006;47:2042-2048.

13. Vilain D, Daou D, Casset-Senon D, Faraggi M, Le GD. Optimal 3-dimensional method for right and left ventricular Fourier phase analysis in electrocardiographygated blood-pool SPECT. J Nucl Cardiol. 2001;8:371-378.

14. Germano G, Erel J, Lewin H, Kavanagh PB, Berman DS. Automatic quantitation of regional myocardial wall motion and thickening from gated technetium- $99 \mathrm{~m}$ sestamibi myocardial perfusion single-photon emission computed tomography. J Am Coll Cardiol. 1997;30:1360-1367.

15. Chen J, Garcia EV, Folks RD, et al. Onset of left ventricular mechanical contraction as determined by phase analysis of ECG-gated myocardial perfusion SPECT imaging: development of a diagnostic tool for assessment of cardiac mechanical dyssynchrony. J Nucl Cardiol. 2005;12:687-695.

16. Garcia EV, Faber TL, Cooke CD, Folks RD, Chen J, Santana C. The increasing role of quantification in clinical nuclear cardiology: the Emory approach. $\mathrm{J} \mathrm{Nucl}$ Cardiol. 2007; 14:420-432.

17. Germano G, Kavanagh PB, Slomka PJ, Van Kriekinge SD, Pollard G, Berman DS. Quantitation in gated perfusion SPECT imaging: the Cedars-Sinai approach. J Nucl Cardiol. 2007; 14:433-454.

18. Van Kriekinge SD, Nishina H, Ohba M, Berman DS, Germano G. Automatic global and regional phase analysis from gated myocardial perfusion SPECT imaging: application to the characterization of ventricular contraction in patients with left bundle branch block. J Nucl Med. 2008;49:1790-1797.

19. Rector TS, Kubo SH, Cohn JN. Validity of the Minnesota Living with Heart Failure Questionnaire as a measure of therapeutic response to enalapril or placebo. Am J Cardiol. 1993;71:1106-1107.
20. Cerqueira MD, Weissman NJ, Dilsizian V, et al. Standardized myocardial segmentation and nomenclature for tomographic imaging of the heart: a statement for healthcare professionals from the Cardiac Imaging Committee of the Council on Clinical Cardiology of the American Heart Association. Int J Cardiovasc Imaging. 2002;18:539-542.

21. Germano G, Kiat H, Kavanagh PB, et al. Automatic quantification of ejection fraction from gated myocardial perfusion SPECT. J Nucl Med. 1995;36:21382147.

22. O'Connell JW, Schreck C, Moles M, et al. A unique method by which to quantitate synchrony with equilibrium radionuclide angiography. J Nucl Cardiol. 2005; 12:441-450.

23. Kapetanakis S, Kearney MT, Siva A, Gall N, Cooklin M, Monaghan MJ. Realtime three-dimensional echocardiography: a novel technique to quantify global left ventricular mechanical dyssynchrony. Circulation. 2005;112:992-1000.

24. Schiller NB, Shah PM, Crawford M, et al. Recommendations for quantitation of the left ventricle by two-dimensional echocardiography. American Society of Echocardiography Committee on Standards, Subcommittee on Quantitation of Two-Dimensional Echocardiograms. J Am Soc Echocardiogr. 1989;2: 358-367.

25. Bleeker GB, Kaandorp TA, Lamb HJ, et al. Effect of posterolateral scar tissue on clinical and echocardiographic improvement after cardiac resynchronization therapy. Circulation. 2006;113:969-976.

26. Bleeker GB, Schalij MJ, Molhoek SG, et al. Relationship between QRS duration and left ventricular dyssynchrony in patients with end-stage heart failure. J Cardiovasc Electrophysiol. 2004;15:544-549.

27. Trimble MA, Borges-Neto S, Smallheiser S, et al. Evaluation of left ventricular mechanical dyssynchrony as determined by phase analysis of ECG-gated SPECT myocardial perfusion imaging in patients with left ventricular dysfunction and conduction disturbances. J Nucl Cardiol. 2007;14:298-307.

28. Trimble MA, Velazquez EJ, Adams GL, et al. Repeatability and reproducibility of phase analysis of gated single-photon emission computed tomography myocardial perfusion imaging used to quantify cardiac dyssynchrony. Nucl Med Commun. 2008;29:374-381.

29. Henneman MM, Chen J, Ypenburg C, et al. Phase analysis of gated myocardial perfusion single-photon emission computed tomography compared with tissue Doppler imaging for the assessment of left ventricular dyssynchrony. J Am Coll Cardiol. 2007;49:1708-1714.

30. Marsan NA, Henneman MM, Chen J, et al. Real-time 3-dimensional echocardiography as a novel approach to quantify left ventricular dyssynchrony: a comparison study with phase analysis of gated myocardial perfusion single photon emission computed tomography. J Am Soc Echocardiogr. 2008;21:801807.

31. Henneman MM, Chen J, Dibbets-Schneider P, et al. Can LV dyssynchrony as assessed with phase analysis on gated myocardial perfusion SPECT predict response to CRT? J Nucl Med. 2007;48:1104-1111.

32. Faber TL, Cooke CD, Folks RD, et al. Left ventricular function and perfusion from gated SPECT perfusion images: an integrated method. J Nucl Med. 1999; 40:650-659.

33. White JA, Yee R, Yuan X, et al. Delayed enhancement magnetic resonance imaging predicts response to cardiac resynchronization therapy in patients with intraventricular dyssynchrony. J Am Coll Cardiol. 2006;48:1953-1960.

34. Adelstein EC, Saba S. Scar burden by myocardial perfusion imaging predicts echocardiographic response to cardiac resynchronization therapy in ischemic cardiomyopathy. Am Heart J. 2007;153:105-112.

35. Murphy RT, Sigurdsson G, Mulamalla S, et al. Tissue synchronization imaging and optimal left ventricular pacing site in cardiac resynchronization therapy. Am J Cardiol. 2006;97:1615-1621.

36. Ypenburg C, van Bommel RJ, Delgado V, et al. Optimal left ventricular lead position predicts reverse remodeling and survival after cardiac resynchronization therapy. J Am Coll Cardiol. 2008;52:1402-1409. 